\title{
Analysis of Pseudomonas fluorescens inoculation effect on the work of mycorrhiza formed on black medic by arbuscular fungi differing in symbiotic activity \\ Zheleznyakov S.V., Lebedeva V.K., Kalinina T.V., Kozhemyakov A.P., Jacobi L.M. \\ ARRIAM, Saint Petersburg, Russia \\ krosh15_02@mail.ru
}

Key message. The influence of rhizobacteria Pseudomonas fluorescens, as well as four species of fungi from the Glomeromycota phylum on the productivity of black medic in mono - and double (fungus + bacteria) inoculation was studied. A high dependence of the results on the symbiotic activity of mycosymbiont was established.

Keywords: arbuscular mycorrhizal fungi (AMF), Pseudomonas fluorescens, mono- and double inoculations, black medic (Medicago lupulina L. subsp. vulgaris Koch.)

Creating the conditions for the effective operation of arbuscular mycorrhiza (AM) is the key to improving the phosphorus nutrition of agricultural plants and increasing their productivity. It is known that the efficiency of AM depends on the species of mycosymbiont. In addition, it is known about the positive effect of bacterial preparations of fertilizer action on plant productivity. The conditions for the stable synergistic effect from the interaction of selected rhizobacteria with AMF on plant productivity have not been studied enough. The solution to this problem is an important stage both for understanding the mechanisms involved in the regulation of AM efficacy and for realization of practical tasks related to increasing the productivity of agricultural plants by mobilizing natural resources.

The effect of inoculation by $P$. fluorescens strain PG7 on the productivity of black medic during the formation of AM with four species of AMF, such as Funneliformis mosseae (AMF-1), G. sp. (AMF-2), Rhizophagus irregularis (AMF-3) and R. diaphanus (AMF-4) differing in symbiotic activity was studied in the conditions of vegetation experiment. Plants were grown on the sterilized soil - sand substrate with the content of available for plant nutrient phosphorus (P) $7.2 \mathrm{mg} \mathrm{P}_{2} \mathrm{O}_{5}$ per $100 \mathrm{~g}$. To bring the soil conditions closer to natural ones, an aqueous suspension of microorganisms, without propagules of AMF, obtained from the unsterilized soil by filtration through a thin mill sieve was added to the substrate. Plant seedlings were inoculated with microorganisms when planting in the soil by introducing the mycorrhizal roots of Plectranthus australis and the aqueous suspension of bacteria.

The results of the analysis carried out on the 54th day from the planting of seedlings showed that in the absence of AM, inoculation by P. fluorescens did not affect the plant growth. Whereas, the effect of AM on plant growth in the absence of inoculation by $P$. fluorescens largely depended on the AMF species. For example, inoculation with inactive AMP-1 had a significant negative effect on root growth (33\% weight loss) and did not affect the yield of straw and the content of total $\mathrm{P}$ in it. Under conditions of inoculation with a medium-active AMF-2 strain, the yield of roots and straw was at the control level; and only a significant increase of the total $\mathrm{P}$ in straw (by 66.5\%) indicated the symbiotic activity of AMF-2. The inoculation by an active AMF-3 and AMF-4 strains had a significant positive impact on the growth of the aerial parts (increase by $36 \%$ in both cases) and the content of total $\mathrm{P}$ in straw (an increase of $81.1 \%$ and $70.6 \%$, respectively) and did not affect the growth of roots compared with the control (without AM).

The effect of double inoculation (fungus + bacteria) on plant growth depended on the mycosymbiont species. A significant increase in mycorrhizal formation was observed in the conditions of double inoculation of $P$. fluorescens and AMF-1 in comparison with monoinoculation by AMF-1. However, this did not have a significant effect on plant productivity compared to the control (AMF-1 monoinoculation). The double inoculation by AMF-2 and P. fluorescens revealed a synergistic effect on plant growth: significantly increased root weight (by 33.1\%) and straw yield (by 35.3\%) compared to the control (AMF-2 monoinoculation). The double inoculation by $P$. fluorescens and AMF-3 did not lead to significant changes in in plant productivity. The double inoculation by P. fluorescens and AMF-4 had a significant synergistic effect on plant growth: straw yield increased by $22.7 \%$, root weight by $19.6 \%$ compared to the control (AMF-4 monoinoculation). In this case the highest yield of straw was obtained.

Thus, the effectiveness of inoculation of highly mycotrophic black medic by $P$. fluorescens PG7 largely depended on both the presence of $\mathrm{AM}$ and the species of $\mathrm{AMF}$ in the inoculum.

This work was supported by the RFBR grant №19-016-00197. 